\title{
Triglyceride regulate ACE2 level through MTHFD1
}

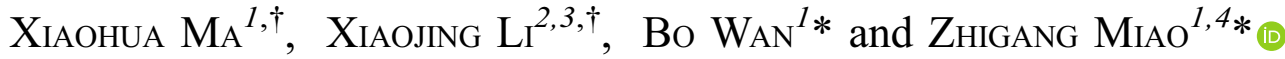 \\ ${ }^{1}$ Institute of Neuroscience, Soochow University, Suzhou, China \\ ${ }^{2}$ Department of Neurology, Suzhou Hospital Affliated to Nanjing Medical University, Suzhou, \\ Jiangsu, China \\ ${ }^{3}$ Department of Neurology, Suzhou Science and Technology Town Hospital, Suzhou, Jiangsu, China \\ ${ }^{4}$ Jiangsu Key Laboratory of Neuropsychiatric Diseases, Soochow University, Suzhou 215123, \\ Jiangsu, China
}

*Corresponding authors (Emails, bowan@fudan.edu.cn; zgmiao@suda.edu.cn)

${ }^{\dagger}$ Xiaohua Ma and Xiaojing Li are Co-first authors.

MS received 12 November 2020; accepted 18 May 2021

\begin{abstract}
Obesity has been followed with interest as a risk factor for COVID-19, with triglycerides as one of four common criteria used to define obesity, which have been used to study the mechanism of obesity. In this study, we showed that angiotensin-converting enzyme-2 (ACE2) is widely expressed in the mouse body, including the kidney, spleen, brain, heart, lung, liver, and testis, and that ACE2 levels increased after a high-fat diet. The ACE2 levels were recorded at 0 days, 3 days, 7 days, and 14 days after a high-fat diet, and they increased at 14 days after high-fat diet initiation. In addition, triglyceride levels were also significantly increased at 14 days after high-fat diet initiation, but body weight was not changed. Furthermore, we examined the ACE2 levels in Calu3 cells (a lung cancer cell line) after triglyceride treatment, and the results indicated that ACE2 levels were increased at $25 \mu \mathrm{M}$ and reached their peak at $200 \mu \mathrm{M}$. Finally, we found that the mRNA level of mthfd1 was significantly increased in the high-fat diet group. Given these findings, we hypothesize that triglycerides can regulate the expression of ACE2 and Mthfd1.
\end{abstract}

Keywords. Triglyceride; COVID-19; Obesity; ACE2; Mthfd1

\section{Introduction}

The COVID-19 pandemic is bringing public health to the forefront, and it is thought to be related to the SARS virus. Currently, more than 5 million people have contracted COVID-19, and cumulative deaths exceed 340,000 (WHO Coronavirus Disease (COVID19) Dashboard, published 24 May 2020). There are many risk factors for virus infection, and an increasing number of studies have found that obesity is one of the major risk factors for COVID-19 (Kassir 2020; Lighter et al. 2020; Zhang et al. 2020).

Triglycerides (TGs) are mainly synthesized in the liver and adipose tissue, often as biomarkers for obesity. Following an increasing number of studies, TGs have been reported to play an important role in many diseases, including those in various tissues and organs, such as the brain(Croteau et al. 2018), heart (Goldberg 2018), liver (Alves-Bezerra and Cohen 2017), lung (Zuber et al. 2016), kidney (Bobulescu et al. 2014), spleen (Portois et al. 2007), and testis (Wang et al. 2019). We know that SARS-CoV-2 not only causes lung injury but also causes complications in most other organs, and additional studies have found that obesity increases the mortality of infected people and serves as one of the risks of viral infection (Jose and Manuel 2020; Peng et al. 2020; Simonnet et al. 2020; Zheng et al. 2020). Thus, we hypothesize that TGs may be involved in these processes, which will contribute to research on the mechanism of the novel coronavirus.

Angiotensin-converting enzyme-2 (ACE2) receptors are widely expressed in the heart, vessels, gut, lung, testis, and brain and are also present in lung alveolar epithelial cells, small intestine enterocytes, arterial and 
venous endothelial cells and arterial smooth muscle cells (Harmer et al. 2002; Hamming et al. 2004). The ACE2 structure includes an N-terminal and a C-terminal domain with a single transmembrane helix and an intracellular segment (Ye et al. 2017). The pathological function of ACE2 is to mediate entry into the cells of some coronaviruses (Verdecchia et al. 2020). Recently, ACE2 receptors have been shown to be functional receptors for SARS-CoV-2 to enter host target cells (Kai and Kai 2020). SARS-CoV-2, through the viral structural spike (S) protein, binds to the ACE2 receptor to target cells (Sanders, Monogue et al. 2020). The mechanism is that SARS-CoV-2 attaches to cells through ACE2. Then, the type II transmembrane protease serine 2 (TMPRSS2) processes SARS-CoV-2-S to expose a cell-membrane fusion peptide. Once membrane fusion occurs, viral RNA is released into the infected host cell and begins viral replication (Nitulescu et al. 2020). Currently, it has been proposed that inhibiting this interaction might be used in treating patients with COVID-19 (Jackson et al. 2020). In this study, we will study the relationship between triglycerides and ACE2 in mice and cells to explain the specific mechanism by which obesity leads to increased viral infections.

\section{Materials and methods}

\subsection{Animals and cells}

Male C57BL/6 mice were purchased from SLAC Company (Shanghai, China) in this study. All procedures performed in these studies involving animals were in accordance with the ethical standards of the University Committee on Animal Care of Soochow University and the Guidelines of Animal Use and Care of the National Institutes of Health (NIH). The number and date for ethical approval was 202005A033. Calu3 cells (ATCC: HTB-55) were purchased from FuHeng BioLogy Company (Shanghai, China).

\subsection{Western blot analysis}

After high-fat diet consumption, the whole lungs of mice were collected, ground in liquid nitrogen and stored at $-80^{\circ} \mathrm{C}$. Approximately $100 \mathrm{mg}$ of tissue was added to $200 \mu \mathrm{l}$ of lysis buffer for $30 \mathrm{~min}$ on ice and centrifuged for $30 \mathrm{~min}$ at $4{ }^{\circ} \mathrm{C}$ and $16,000 \times \mathrm{g}$. The supernatants were mixed with loading buffer and boiled for $10 \mathrm{~min}$ at $96^{\circ} \mathrm{C}$ for Western blot analysis after cooling. Approximately $30 \mu \mathrm{g}$ samples were separated by sodium dodecyl sulfate-polyacrylamide gel electrophoresis and then transferred to nitrocellulose membranes. Membranes were blocked, incubated with the primary antibody, incubated with the secondary antibody, and then developed. The densitometry of the bands was analysed with Alpha Ease Image Analysis Software V3.1.2. Anti-ACE2 (CY5787) antibodies were purchased from Abways Technology Company (Shanghai, China); mouse monoclonal antiActin (ER62585) antibodies were purchased from HUABIO Company (Hangzhou, China).

\subsection{Reverse transcription polymerase chain reaction}

Total RNA was extracted from lung tissues. Reverse transcription of cDNA was conducted with SYBR green for reverse transcription PCR (RT-PCR). GAPDH (forward: CATGGCCTTCCGTGTTCCTA; reverse: CTTCACCACCTTCTTGATGTCATC) was used as a control. Mthfd1 (forward: AGAGGGCGAGCTTTATCACG; reverse: CCAGGTTCCCGATGAGCTTT) was detected.

\subsection{Cell viability and triglyceride detection}

Cell viability was detected by a CCK 8 Assay Kit (CK04, DOJINDO Co., Ltd., Shanghai, China) in a 96-well cell culture plate, $10 \mu \mathrm{l}$ per well was added, and the OD value was measured after $2 \mathrm{~h}$. Serum triglycerides (TGs) in mice were measured by using a Triglyceride Colorimetric Assay Kit (E-BC-K261-M, Elabscience Biotechnology Co., Ltd., Wuhan, China) according to the manufacturer's instructions.

\subsection{Statistical analysis}

All data are expressed as the means \pm SEMs. GraphPad Prism 6 (GraphPad Software Inc., La Jolla, CA, USA) was used for statistical analysis. In figure $3 \mathrm{E}$ Student's $t$-test was used to determine the differences. In figure $2 \mathrm{~A}, \mathrm{~B}$, and $\mathrm{D}$ one-way analysis to compare differences was used. $\mathrm{P}<0.05$ was considered statistically significant. 


\section{Results}

\subsection{Levels of ACE2 in mouse tissues}

Previous studies reported that ACE2 exists in various tissues and cells (Hamming et al. 2004), and we examined the distribution of ACE2 in different tissues of mice by Western blot. We found that ACE2 was highly expressed in the kidney and lowly in the spleen (figure 1A); there was a similar expression level in the brain, heart, lung, liver, and testis (figure 1A). Quantitative analysis confirmed the expression of ACE2 in different tissues (figure 1B).

\subsection{Levels of ACE2 in mouse lung tissue in fat mice}

To reveal the relationship between obesity and ACE2 protein, we selected 12 mice of the same age, and they were divided two groups, high weight and light weight. We tested the TG concentration in the different mice serum, and then took the lung tissue to test the expression of ACE2. The results showed that higher TG concentration in high weight mice (figure 2B), and the expression of ACE2 was also significantly increased in high weight mice (figure 2C and 2D).

To further prove the relationship between TG and ACE2,we established an obese mouse model by using
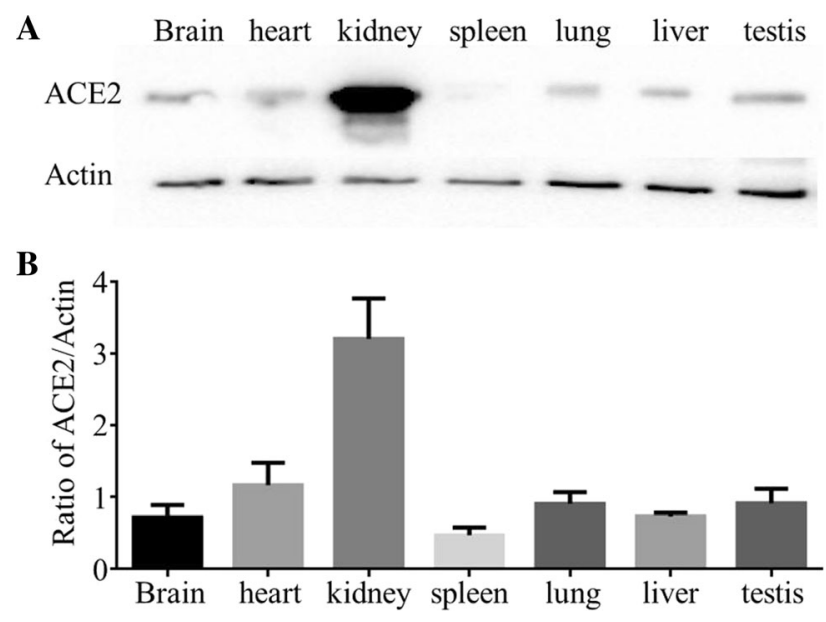

Figure 1. The distribution of ACE2 in mouse tissue. ACE2 levels were determined by Western blot in different tissue of mice. The representative photographs showed ACE2; Actin was used as a loading control (A). The quantitative analysis was performed on the ratio of ACE2 to Actin (B), mean with $\mathrm{SEM}, \mathrm{N}=3$. a high-fat diet (60\% fat) to feed mice and monitored body weight and triglycerides at 3, 7 and 14 days after the high-fat diet initiation. Although body weight did not change after the high-fat diet $(\mathrm{P}=0.1218$, figure $3 \mathrm{~A}$ ), serum triglyceride levels significantly increased at 14 days after high-fat diet initiation $(\mathrm{P}=0.0145$, figure $3 \mathrm{~B})$. To study the relationship between serum triglycerides and ACE2, we recorded ACE2 levels at 0 days, 3 days, 7 days, and 14 days after high-fat diet initiation. ACE2 expression increased at 14 days after high-fat diet initiation (figure 3C-DAYS). These results suggest that triglycerides may be an important factor in regulating the expression of ACE2.

\subsection{Levels of ACE2 in Calu3 cells after triglyceride treatment}

To further prove that the expression of ACE2 was regulated by serum triglycerides, we examined ACE2 levels in Calu3 cells, a lung cancer cell line, after TG treatment. We treated cells with TGs $(25 \mu \mathrm{M}, 50 \mu \mathrm{M}$, $100 \mu \mathrm{M}, 200 \mu \mathrm{M}$, and $400 \mu \mathrm{M})$ and cultured them for 48 hours in an incubator $\left(37^{\circ} \mathrm{C}, 5 \% \mathrm{CO} 2\right)$. First, we detected the expression of ACE2 by Western blot. The results showed that ACE2 levels increased at $25 \mu \mathrm{M}$ and reached their peak at $200 \mu \mathrm{M}$ TG (figures 4A and 3B). Then, a CCK8 Assay Kit was used to test the activity of the cells after TG treatment. The results showed that a low concentration of TGs did not affect cell survival (figure 4C).

\section{4 mRNA levels of Mthfd1 in mouse lung tissue after a high-fat diet}

Previous studies indicate that methylenetetrahydrofolate dehydrogenase (MTHFD1) is a protein that affects immune function and obesity (Christensen et al. 2014; Vohra et al. 2020). To explore the mechanism by which TG leads to increased ACE2, we examined the expression of MTHFD1 by RT-PCR in mice after a high-fat diet. We extracted RNA from mouse lung tissue and detected the level of the mthfdl gene. As shown in figure $4 \mathrm{D}$, the mRNA level of mthfdl was significantly increased in mice fed a high-fat diet compared with that in control mice. The statistical analysis is shown in figure $4 \mathrm{E}(\mathrm{p}<0.05)$. 
A

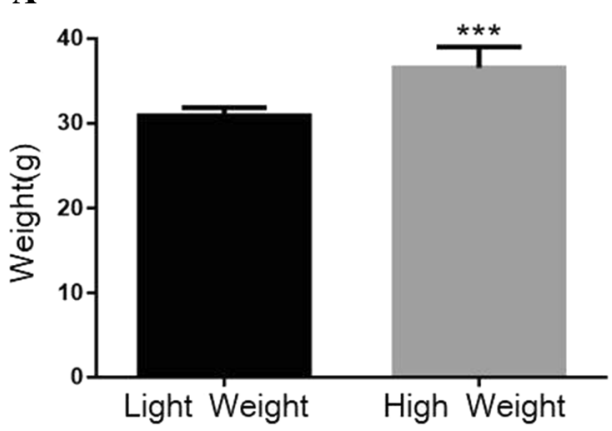

C

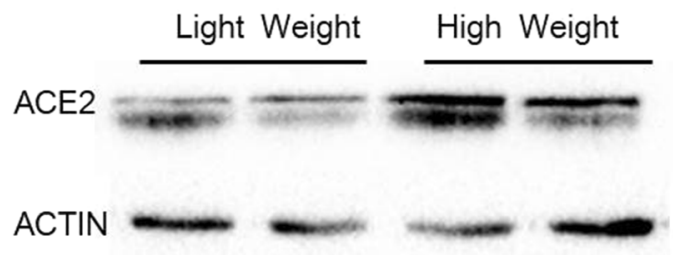

B

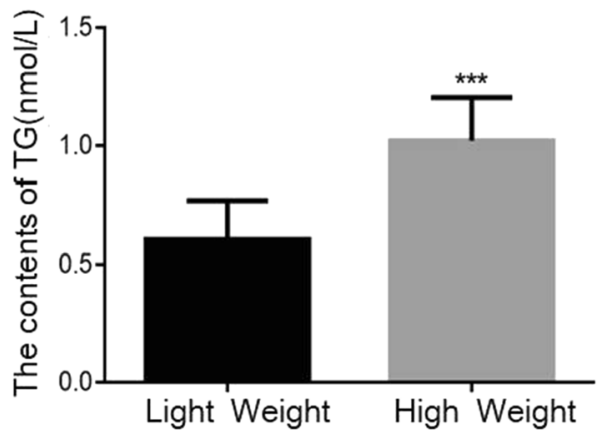

D

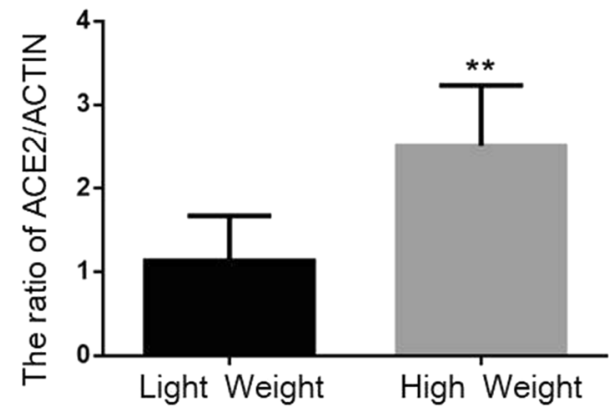

Figure 2. The levels of TG and ACE2 in fat mouse. The same age mice were divided two groups, high weight and light weight. Weight of mice (A). The triglyceride concentration was detected by ELISA kit in mouse serum (B). ACE2 levels were determined by Western blot $(\mathbf{C})$. Quantitative analysis of ACE2 levels was performed (D). Mean with SEM, N=6, $* \mathrm{P}<0.05$, versus the light weight group.

\section{Discussion}

Obesity has become a worldwide health concern because it increases the risk of some diseases (Wang et al. 2011). Scientists predict that $86 \%$ of adults will be overweight or obese in the USA by 2030 (Ginter and Simko 2014). At present, the number of COVID19 viral infections is approximately 160 million people, and the number of deaths is over 3300,000 worldwide; the United States has the largest number of infections, which may be related to its high obesity rate. Most studies have confirmed that obesity can aggravate the condition of COVID-19 patients and increase the fatality rate (Finer et al. 2020; Kassir 2020; Stefan et al. 2020). Metabolically healthy obese (MHO) sets four common criteria used to define obesity, including blood pressure, HDL cholesterol, triglycerides, and plasma glucose (Bergman et al. 2011). Hypertriglyceridemia is the most prevalent metabolic change in obesity (Guijarro de Armas et al. 2012). Our results showed that the concentration of TGs was significantly increased in high weight mice (figure 2B) and high-fat diet (figure 3B), in accordance with the symptoms of obesity, although the body weight of mice did not change (figure 3A). Gillian et al. (Rice et al. 2006) showed a correlation between ACE2 and triglyceride levels, and our results confirmed that high triglycerides can cause ACE2, a protein that promotes the entry of viruses into cells, to be significantly increased in animal experiments (Figures 2C and 3C). In addition, ACE2 levels were significantly increased in Calu3 cells compared with those in control cells after $\mathrm{TG}$ treatment (figure 4A). Our results suggested that more serious illness may be caused by increased expression of ACE2 in high-triglyceride people with COVID-19.

ACE2 consists of an 805 amino acid type I transmembrane glycoprotein and contains a short intracellular cytoplasmic tail and a longer extracellular domain (Lambert et al. 2010). ACE2 was reported to be a potential receptor in infections with severe acute respiratory syndrome coronavirus (SARS-CoV) in 2003 (Li et al. 2003; Peiris et al. 2003). The mechanism is that SARS infections are initiated by the binding of the spike protein trimers of SARS-CoV to a hydrophobic pocket of the extracellular catalytic domain of ACE2 (Li et al. 2005). The novel coronavirus is a type of coronavirus that also plays an important role. Methylenetetrahydrofolate dehydrogenase 1 (MTHFD1) 

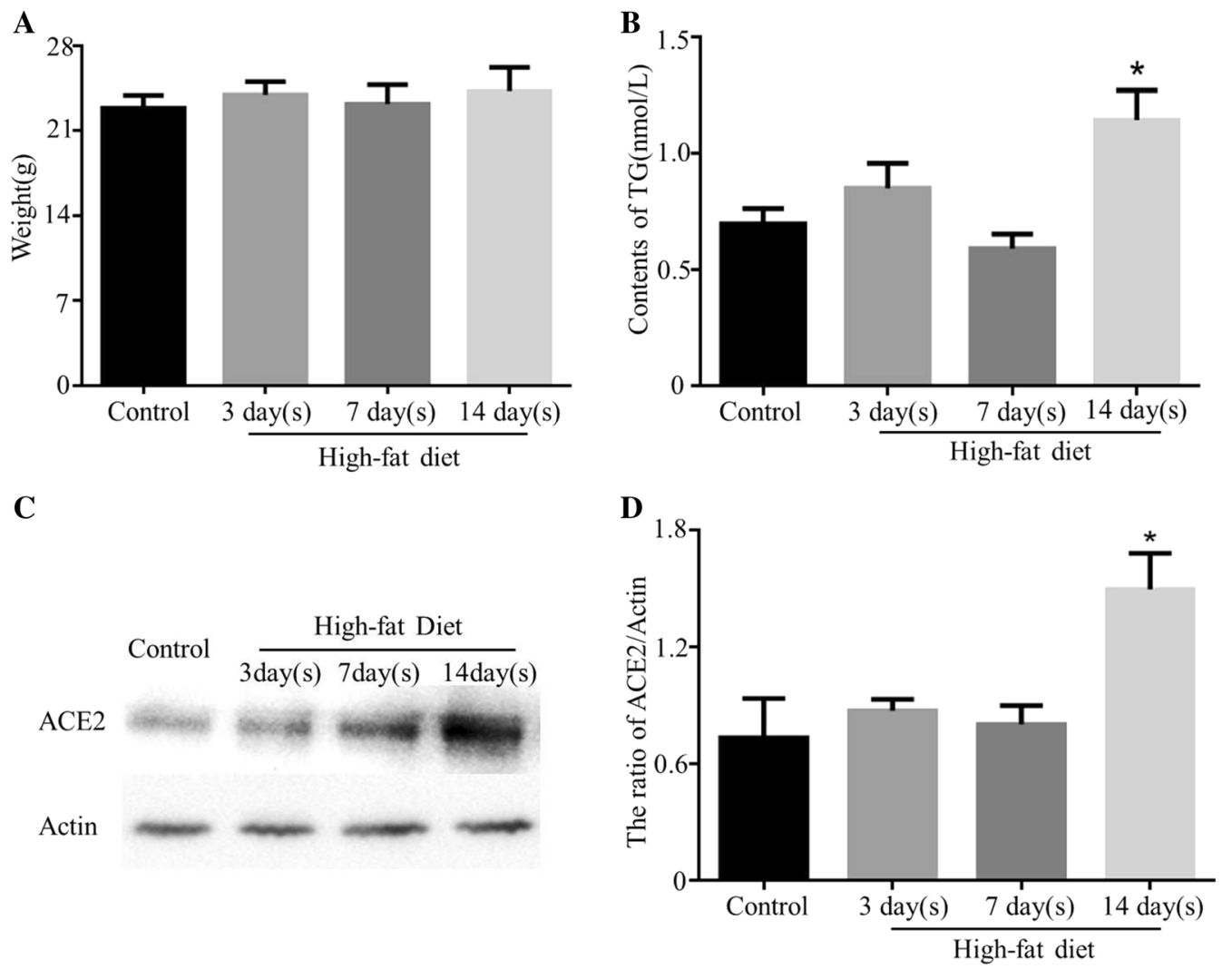

Figure 3. The levels of ACE2 in mouse lung tissue after the high-fat diet. Mice were fed with high-fat diet 3 days, 7 days, and 14 days, the control group was feed with Normal diet. The weight of mice was examined after high-fat diet. The representative photograph (A). The concentration of TG was tested after High-fat Diet. Representative photograph (B). ACE2 levels were evaluated according to Western blot $(\mathbf{C})$ and the quantitative analysis was performed on the ratio of ACE2 to Actin (D). Mean with SEM, N=6, * $\mathrm{P}<0.05$, versus the control group.

is a pivotal enzyme in the folic acid metabolic pathway. It can regulate nucleotide formation and DNA methylation. The average global DNA methylation was reduced by $40 \%$ in the mthfd 1 mutant phenotype compared with that in the WT, and MTHFD1 is required for epigenetic silencing (Groth et al. 2016). Obesity may also induce alterations in DNA methylation signatures in offspring (Samblas et al. 2019), and saturated fatty acids induce some gene hypermethylation, increasing or decreasing the expression of these genes (Flores-Sierra et al. 2016). In our studies, we found that the mRNA level of Mthfd1 was significantly increased after the high-fat diet (figure 4D), and we hypothesize that triglycerides regulate the expression of ACE2 through Mthfd1 and influence DNA methylation. Notably, this hypothesis needs additional experimental research to confirm. In the future, we will examine the transcription and protein expression levels of ACE2 after overexpression and knockout of MTHFD1 in cells, and clarify the relationship between ACE2 and Mthfd1. According to previous studies, we know that both triglycerides (Yu et al. 2018) and mthfd1 (Groth et al. 2016) are related to DNA methylation, we will test the levels of DNA methylation after used ACE2 inhibitors or Mthfd1 inhibitors under the TG treatment, to conform that TG regulates the expression of Ace 2 through Mthfd1 affects the methylation levels of Ace 2 gene.

In summary, we showed that the expression of ACE2 is closely related to the triglyceride content. Indepth studies have found that triglycerides can regulate the expression of Mthfd1, and previous studies have reported that Mthfd1 can affect DNA methylation. Therefore, we speculate that triglycerides can 
A

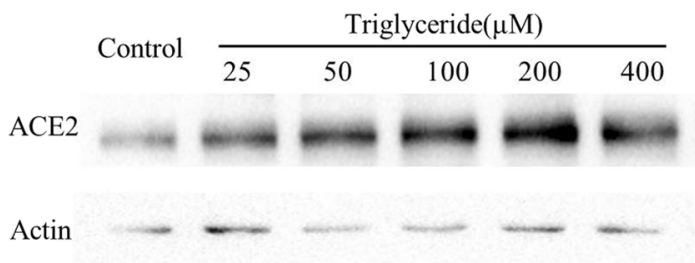

B

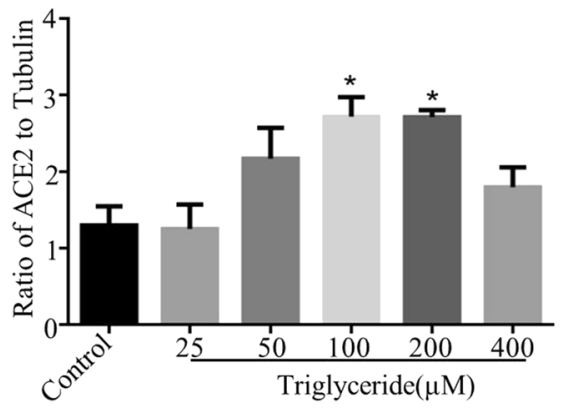

$\mathbf{E}$
C

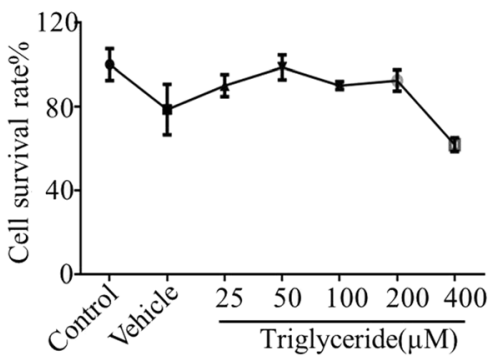

D

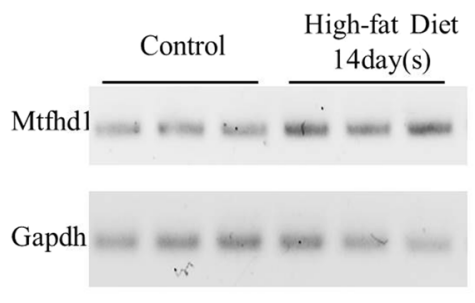

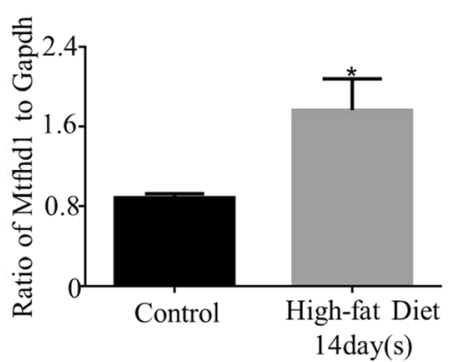

Figure 4. The levels of ACE2 in Calu3 cell after triglyceride treatment and the mRNA levels of Mtfhd1 in mouse lung tissue after high-fat diet. Calu3 cells were administered with TG (25, 50, 100, 200, and $400 \mu \mathrm{M}) 48 \mathrm{~h}$, ACE2 levels were determined by Western blot (A). Quantitative analysis of ACE2 levels was performed (B). The cell survival rate was showed after TG treatment $(\mathbf{C})$. The mRNA level of Mtfhd1 was detected by RT-PCR in fat mice (with high-fat diet 14 days). The representative photograph was shown (D) and the quantitative analysis was performed on the ratio of Mtfhd1 to Gapdh (E). Mean with $\mathrm{SEM}, \mathrm{N}=6, * \mathrm{P}<0.05$, versus the control group.

affect the expression of mthfd1 and regulate the ACE2 protein level by affecting the methylation of the Ace 2 gene.

\section{Acknowledgements}

This study was supported by the grants the National Natural Science Foundation of China (81601154), Science and Technology to people's livelihood Program of Suzhou (Grant no. sys2018012), Jiangsu Key Laboratory of Neuropsychiatric Diseases (BM2013003) and the Priority Academic Program Development of Jiangsu Higher Education Institutions of China.

\section{References}

Alves-Bezerra M and Cohen DE 2017 Triglyceride metabolism in the liver. Compr. Physiol. 8 1-8

Bergman RN, Stefanovski D, Buchanan TA, Sumner AE, Reynolds JC, et al. 2011 A better index of body adiposity. Obesity 19 1083-1089

Bobulescu IA, Lotan Y, Zhang J, Rosenthal TR, Rogers JT, et al. 2014 Triglycerides in the human kidney cortex: relationship with body size. PLoS One 9 e101285
Christensen KE, Dahhou M, Kramer MS and Rozen R 2014 The MTHFD1 $1958 \mathrm{G}>\mathrm{A}$ variant is associated with elevated C-reactive protein and body mass index in Canadian women from a premature birth cohort. Mol. Genet. Metab. 111 390-392

Croteau E, Castellano CA, Richard MA, Fortier M, Nugent $\mathrm{S}$, et al. 2018 Ketogenic medium chain triglycerides increase brain energy metabolism in Alzheimer's disease. J. Alzheimers Dis. 64 551-561

Finer N, Garnett SP and Bruun JM 2020 COVID-19 and obesity. Clin. Obes. 10 e12365

Flores-Sierra J, Arredondo-Guerrero M, Cervantes-Paz B, Rodriguez-Rios D, Alvarado-Caudillo Y, et al. 2016 The trans fatty acid elaidate affects the global DNA methylation profile of cultured cells and in vivo. Lipids Health Dis. 1575

Ginter E and Simko V 2014 Becoming overweight: is there a health risk? Bratisl. Lek. Listy. 115 527-531

Goldberg IJ 20182017 George Lyman Duff Memorial Lecture: Fat in the blood, fat in the artery, fat in the heart: triglyceride in physiology and disease. Arterioscler. Thromb. Vasc. Biol. 38 700-706

Groth M, Moissiard G, Wirtz M, Wang H, Garcia-Salinas C, et al. 2016 MTHFD1 controls DNA methylation in Arabidopsis. Nat. Commun. 711640

Guijarro de Armas MA, Monereo Megias S, Merino Viveros M, Iglesias Bolanos P and Vega Pinero B 2012 Prevalence of 
metabolic syndrome in a population of obese children and adolescents. Endocrinol. Nutr. 59 155-159

Hamming I, Timens W, Bulthuis ML, Lely AT, Navis G, et al. 2004 Tissue distribution of ACE2 protein, the functional receptor for SARS coronavirus. A first step in understanding SARS pathogenesis. J. Pathol. 203 631-637

Harmer D, Gilbert M, Borman R and Clark KL 2002 Quantitative mRNA expression profiling of ACE 2, a novel homologue of angiotensin converting enzyme. FEBS Lett. 532 107-110

Jackson DJ, Busse WW, Bacharier LB, Kattan M, O'Connor GT, et al. 2020 Association of respiratory allergy, asthma and expression of the SARS-CoV-2 receptor, ACE2. $J$. Allergy Clin. Immunol

Jose RJ and Manuel A 2020 Does COVID-19 Disprove the Obesity Paradox in ARDS? Obesity

Kai H and Kai M 2020 Interactions of coronaviruses with ACE2, angiotensin II, and RAS inhibitors-lessons from available evidence and insights into COVID-19. Hypertens Res

Kassir R 2020 Risk of COVID-19 for patients with obesity. Obes. Rev. 21 e13034

Lambert DW, Clarke NE and Turner AJ 2010 Not just angiotensinases: new roles for the angiotensin-converting enzymes. Cell Mol. Life Sci. 67 89-98

Li F, Li WH, Farzan M and Harrison SC 2005 Structure of SARS coronavirus spike receptor-binding domain complexed with receptor. Science 309 1864-1868

Li WH, Moore MJ, Vasilieva N, Sui JH, Wong SK, et al. 2003 Angiotensin-converting enzyme 2 is a functional receptor for the SARS coronavirus. Nature 426 450-454

Lighter J, Phillips M, Hochman S, Sterling S, Johnson D et al. 2020 Obesity in patients younger than 60 years is a risk factor for Covid-19 hospital admission. Clin. Infect. Dis. https://doi.org/10.1093/cid/ciaa415

Nitulescu GM, Paunescu H, Moschos SA, Petrakis D, Nitulescu G, et al. 2020 Comprehensive analysis of drugs to treat SARSCoV2 infection: Mechanistic insights into current COVID19 therapies. Int. J. Mol. Med. 46 467-488

Peiris JS, Yuen KY, Osterhaus AD and Stohr K 2003 The severe acute respiratory syndrome. $N$ Engl J Med 349 2431-2441

Peng YD, Meng K, Guan HQ, Leng L, Zhu RR, et al. 2020 Clinical characteristics and outcomes of 112 cardiovascular disease patients infected by 2019-nCoV. Zhonghua Xin Xue Guan Bing Za Zhi 48 E004

Portois L, Sener A, Carpentier YA and Malaisse WJ 2007 Fatty acid content and pattern of spleen phospholipids and triglycerides in normal and either type-1 or type-2 diabetic rats. Int. J. Mol. Med. 19 523-528

Rice GI, Jones AL, Grant PJ, Carter AM, Turner AJ, et al. 2006 Circulating activities of angiotensin-converting enzyme, its homolog, angiotensin-converting enzyme 2, and neprilysin in a family study. Hypertension $\mathbf{4 8}$ 914-920

Samblas M, Milagro FI and Martinez A 2019 DNA methylation markers in obesity, metabolic syndrome, and weight loss. Epigenetics 14 421-444

Sanders JM, Monogue ML, Jodlowski TZ and Cutrell JB 2020 Pharmacologic treatments for coronavirus disease 2019 (COVID-19): A review. JAMA 323 1824-1836

Simonnet A, Chetboun M, Poissy J, Raverdy V, Noulette J, et al. 2020 High prevalence of obesity in severe acute respiratory syndrome coronavirus-2 (SARS-CoV-2) requiring invasive mechanical ventilation. Obesity

Stefan N, Birkenfeld AL, Schulze MB and Ludwig DS 2020 Obesity and impaired metabolic health in patients with COVID-19. Nat. Rev. Endocrinol. https://doi.org/10. 1038/s41574-020-0364-6

Verdecchia P, Cavallini C, Spanevello A and Angeli F 2020 The pivotal link between ACE2 deficiency and SARSCoV-2 infection. Eur. J. Intern. Med. https://doi.org/10. 1016/j.ejim.2020.04.037

Vohra M, Adhikari P, Souza SC, Nagri SK, Umakanth S et al. $2020 \mathrm{CpG}-\mathrm{SNP}$ site methylation regulates allelespecific expression of MTHFD1 gene in type 2 diabetes. Lab. Invest. https://doi.org/10.1038/s41374-020-0422-7

Wang F, Ren XF, Chen Z, Li XL, Zhu HJ, et al. 2019 The $\mathrm{N}$-terminal His-tag affects the triglyceride lipase activity of hormone-sensitive lipase in testis. J. Cell Biochem. 120 13706-13716

Wang YC, McPherson K, Marsh T, Gortmaker SL and Brown M 2011 Health and economic burden of the projected obesity trends in the USA and the UK. Lancet 378 815-825

WHO Coronavirus Disease (COVID-19) Dashboard 2020 https://covid19.who.int/

Ye YH, Chen K, Jin KK, Zhang YF and Chen L 2017 Progress on surgical treatment for femoral head-preservering in the precollapse stage of femoral head necrosis. Zhongguo Gu Shang 30 287-292

$\mathrm{Yu}$ JC, Hsu FC and Chiu YF 2018 Assessment of fenofibrate-methylation interactions on triglycerides using longitudinal family data. BMC Proc. 1248

Zhang F, Xiong Y, Wei Y, Hu Y, Wang F, et al. 2020 Obesity predisposes to the risk of higher mortality in young COVID-19 patients. J. Med. Virol.

Zheng KI, Gao F, Wang XB, Sun QF, Pan KH, et al. 2020 Obesity as a risk factor for greater severity of COVID-19 in patients with metabolic associated fatty liver disease. Metabolism

Zuber V, Marconett CN, Shi J, Hua X, Wheeler W, et al. 2016 Pleiotropic analysis of lung cancer and blood triglycerides. J. Natl. Cancer Inst 